\title{
Predicting Near-ground Vortex Lifetimes Using Weibull Density Functions
}

\author{
Lawrence A. Berk ${ }^{1}$ and James N. Hallock ${ }^{2}$ \\ U.S. DOT RITA John A. Volpe National Transportation Systems Center, Cambridge, MA 02142
}

\begin{abstract}
To mitigate safety hazards posed by near-ground vortex lateral transport, under instrument flight rules (IFR), parallel runway operations must adopt aircraft spacing standards that often reduce capacity. Once the phenomenon of lateral transport is understood, it is conceivable that, under IFR, new separation standards could restore some runway capacity. In this context, it is of interest to establish the probability that a wake vortex is still surviving in lateral transport $t$ seconds after it began migrating. This paper applies survival data analysis methods to demonstrate how to generate predictions of survival probability that are specific to aircraft type. Modeling assumptions lead to a family of Weibull probability densities, a form that is useful for modeling wind phenomena.
\end{abstract}

\section{Nomenclature}

$\begin{array}{lll}b & = & \text { wingspan } \\ f(t) & = & \text { vortex lifetime density function } \\ F(t) & = & \text { vortex lifetime cumulative distribution function } \\ h(t) & = & \text { vortex lifetime hazard function } \\ i & = & \text { subscript denoting aircraft weight class } \\ j & = & \text { subscript denoting aircraft type } \\ L & = & W / b^{2}, \text { wing loading } \\ P(\bullet) & = & \text { probability that } \bullet \text {, a vortex demise event, occurs } \\ \boldsymbol{r} & = & \text { Cox-Snell residual vector } \\ S(t) & = & \text { vortex lifetime survival function } \\ t & = & \text { time } \\ T & = & \text { linking time } \\ V & = & \text { random variable for vortex longevity } \\ W & = & \text { maximum landing weight } \\ \boldsymbol{x}_{j} & = & \text { vector of aircraft type } j \text { characteristics } \\ \alpha & = & \text { Weibull density scale parameter } \\ \beta & = & \text { vector of regression coefficients for } \boldsymbol{x} \\ \gamma & = & \text { Weibull density shape parameter } \\ \Delta t & = & \text { time step } \\ \varepsilon & = & \text { random error term } \\ \tau & = & \text { regression coefficient for } \varepsilon\end{array}$

I. Introduction

Vortex survival data by aircraft type was collected at Frankfurt/Main (FRA) International Airport between November 1997 and June 1998; a total of 10,000 vortex lifetime measurements were made by an anemometer array positioned at the eastern end of the parallel runways (Fig. 1). ${ }^{1}$ Since the purpose of this study is to produce aircraftspecific Weibull probability densities, during data preparation care was taken to estimate aircraft attributes (e.g., wingspan or landing weight) based only on aircraft variants in service at the time of the Frankfurt experiment. ${ }^{2}$

\footnotetext{
${ }^{1}$ Analyst, Safety Information Systems Division, RTV-5B

${ }^{2}$ Senior Technical Expert for Air and Space Transportation Safety, RTV-5B, Senior Member AIAA
} 


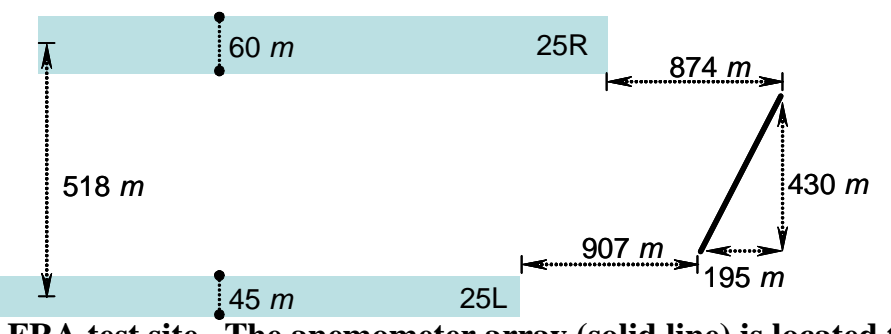

Figure 1. The FRA test site. The anemometer array (solid line) is located to the east.

Large eddy simulation (LES) suggests average circulation decay is not a linear function, but rather follows a distinctive two-phase process. Beyond a linking time $T$, average circulation decays more rapidly. ${ }^{3,4}$ Prior to $T$ vortices retain much of their initial strength and this slow decay in vortex strength suggests vortex longevity probabilities also decline slowly for $0<t<T$. Likewise, vortex demise soon after $T$ (rapid loss of strength) also suggest survival probabilities fall at a much higher rate when $t>T$. In summary, as vortices age, LES suggests the probability of demise per unit time is also non-linear (and increasing). ${ }^{4}$ This conjecture motivates a survival data analysis of $V$, a random variable for vortex longevity.

For any survival process described by a probability density, a hazard function expresses the risk of demise per unit time. This paper illustrates that modeling $V$ with two-parameter Weibull densities $-V \sim \operatorname{Weibull}(\alpha, \gamma)-$ accurately estimates vortex longevity distributions. A data set comprising 10,000 vortex lifetime measurements at Frankfurt am Main International Airport offers evidence that hazard functions of near-ground vortices are nearly quadratic. Such polynomial hazard functions are, by definition, associated with Weibull density functions. ${ }^{5}$

A salient result of this survival data analysis is a set of aircraft-specific density functions for vortex longevity. Model fit and diagnostics produce a set of $V \sim$ Weibull $(\alpha, \gamma)$ with scale parameters $(\alpha)$ that are linear combinations of aircraft characteristics, and shape parameters $(\gamma)$ that are strictly time-dependent. Section II describes details of hazard functions, the Weibull $(\alpha, \gamma)$ density, and the survival analysis of $V$. Section III details Frankfurt data. Sections IV and V interpret and evaluate density functions predicting vortex survival time, by aircraft type, then suggests further research. Finally, the Appendix reviews an earlier regression assessment and why it apparently underestimated Weibull shape parameters $\gamma$.

\section{Modeling Survival Probabilities}

\section{A. The hazard function}

Important to survival data analysis, the probability of demise per unit time is given by the hazard function $h(t)$. The conditional failure rate is another definition of $h(t)$; assuming survival to time $t$, what is the probability of

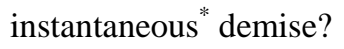

To define $h(t)$ for a random variable $V$, denote the probability density by $f(t)=P(V=t)$ and the cumulative distribution function by $F(t)=P(V \leq t)$. The hazard function for $V$ is the ratio of the probability density $f(t)$ to the survival function $S(t)=P(V>t)=1-F(t)$ :

$$
h(t)=f(t) / S(t)=-\frac{d}{d t} \ln (S(t)) .
$$

The second equality - a consequence of the Chain Rule of calculus ${ }^{\dagger}$ - motivates many methods for exploratory survival data analysis and modeling diagnostics. Note that knowledge of any one of $f(t), S(t)$ or $h(t)$ uniquely determines the other two probability functions.

Equation (1) does not restrict the shape of $h(t)$; depending on the survival process, $h(t)$ may be constant, or it may resemble a ‘bathtub' curve. LES studies suggest vortex circulation decay is a nonlinear process. Prior to a linking time $T$ vortex strength decays slowly. In other words for $0<t<T$ vortices generally preserve their strength as they age, which suggests that vortices are more likely to extend their life beyond $t$. As time passes $S(t)$ decays $S(t)=P(V>t)$ gets less probable for larger $t$ - and beyond $T$ decay occurs more rapidly. The probability of instantaneous demise always increases because the vortex is aging, yet demise probabilities increase at a slower rate between time $0<t<T$.

${ }^{*}$ Defined as demise within the infinitesimally small interval $(t, t+\Delta t)$.

${ }^{\dagger}$ For a differentiable function $g(x), \frac{d}{d x} \ln (g(x))=\frac{1}{g(x)} \cdot\left[\frac{d}{d x} g(x)\right]$. 
Once $T$ is reached, LES suggests vortices do not survive much longer. ${ }^{4}$ If vortex longevity does reach $t>T$, the probability of instantaneous demise increases at a faster rate than it did prior to $T$. In summary, LES results are evidence that an appropriate density function for vortex lifetime $V$ would have an associated nonlinear hazard function $h(t)$. Many density functions possess this property, ${ }^{\ddagger}$ and the assumption $V \sim$ Weibull $(\alpha, \gamma)$ yields accurate predictions and desirable diagnostics.

\section{B. The Weibull distribution}

The Weibull density formula involves a scale parameter $\alpha$ and a shape parameter $\gamma$ :

$$
f(t)=\alpha \gamma t^{\gamma-1} \exp \left(-\alpha t^{\gamma}\right), t \geq 0, \alpha, \gamma>0
$$

The survival function is $S(t)=\exp \left(-\alpha t^{\gamma}\right)$ and, from Eq. (1), the hazard function is

$$
h(t)=-\frac{d}{d t} \ln (S(t))=-\frac{d}{d t}\left(-\alpha t^{\gamma}\right)=\alpha t^{\gamma-1}, t>0 .
$$

Note that $f(t)$ is an Exponential $(\alpha)$ distribution if shape parameter $\gamma=1$, with a constant hazard function $h(t)=\alpha$. Because $h(t)$ is non-constant for $\gamma>1$, Weibull densities generalize the Exponential $(\alpha)$ density. If shape parameter $\gamma$ $=2$ then $h(t)=\alpha \gamma t$ is a linear function of longevity. If $\gamma>2$ then $h(t)=\alpha \gamma t^{\gamma-1}$ is polynomial (monotonically increasing because $t \geq 0$ ). The circulation decay process illustrated by LES data suggests a nonlinear hazard function, so that assuming $V \sim \operatorname{Weibull}(\alpha, \gamma)$ suggests shape parameters $\gamma>2$ (Fig. 2). A monotonically increasing hazard function is a natural consequence of the aging process (as vortices age, instantaneous demise is always more probable).

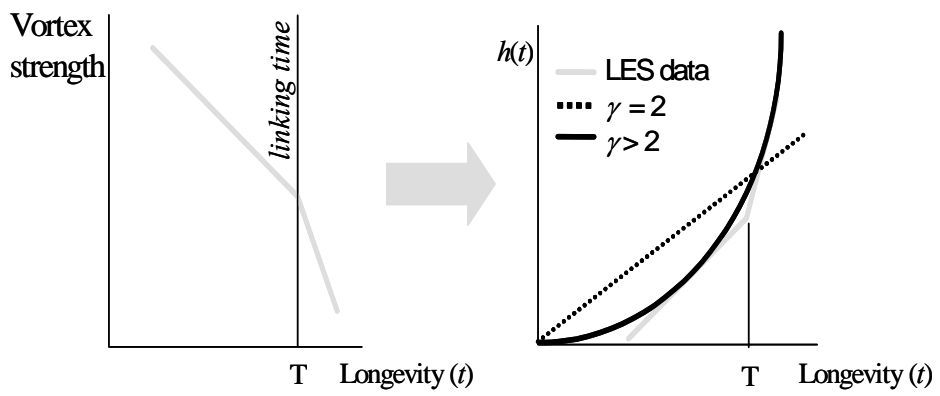

Figure 2. Simplified plots: LES results (left) suggests hazard rates are polynomial, not linear (right).

Vortices most likely to migrate near the other parallel runway were singled out for measurement at Frankfurt airport. Only vortices reaching the second anemometer were measured (by requiring an ambient crosswind of at least $1 \mathrm{~m} / \mathrm{s}$ ) and vortex-induced crosswinds had to be at least $4 \mathrm{~m} / \mathrm{s}$ stronger than the ambient crosswind to ensure detection. The experiment equated demise with a vortex signal no greater than the ambient wind. This favors longer-lived vortices and skews the probability density. ${ }^{1}$ The experimental design might skew probabilities in favor of longer lifetimes (so that a symmetric density may not be a valid assumption). The Weibull shape parameter $\gamma$ quantifies skewness, and estimating it would be beneficial.

\section{Weibull regression}

The method applied by this study assumes a linear relationship between aircraft attributes and the logarithm of vortex survival times (survival analyses refer to this as the accelerated failure time model). For aircraft type $j$, $V_{j} \sim$ Weibull $\left(\alpha_{j}, \gamma\right)$ denotes a random variable of vortex survival and $\boldsymbol{x}_{j}$ denotes attributes of that aircraft. The linear regression model

$$
\ln V_{j}=\beta_{0}+\boldsymbol{\beta} \cdot \boldsymbol{x}_{j}+\tau \cdot \varepsilon_{j}
$$

assumes the independent and identically-distributed random error terms $\varepsilon_{j}$ follow the 'extreme value’ (or Gumbel) distribution, ${ }^{\S}$ not a normal distribution (an assumption of linear least squares regression). ${ }^{5}$ This assumption about

\footnotetext{
${ }^{\ddagger}$ Hazard functions $h(t)$ that depend on $T$ are never assumed.

${ }^{\S}$ For the extreme value distribution (evd), the density is $f(\varepsilon)=\exp (\varepsilon-\exp (\varepsilon))$ and the survival function is $S(\varepsilon)=\exp (-\exp (\varepsilon))$. It turns out that, regardless of the density function assumed, the probability of observing an extreme value (i.e., the minimum or maximum value) among $n$ observations follows an evd, which converges to the
} 
the $\varepsilon_{j}$ implies (see footnote) that $V_{j} \sim$ Weibull $\left(\alpha_{j}, \gamma\right)$ with shape parameter $\gamma \equiv 1 / \tau$ and scale parameter $\alpha_{j}=\exp \left[-\frac{1}{\tau}\left(\beta_{0}+\boldsymbol{\beta} \cdot \boldsymbol{x}_{j}\right)\right]$. Hence this 'Weibull regression' method estimates a set of Weibull distributions $f_{j}\left(t, \boldsymbol{x}_{j}\right)$ possessing a common $\gamma$ yet distinct scale parameters $\alpha_{j}$ (determined by aircraft $j$ attributes) to predict vortex survival time.

Consequently, the survival function $S_{j}\left(t, \boldsymbol{x}_{j}\right)=1-F_{j}\left(t, \boldsymbol{x}_{j}\right)=\exp \left(-\alpha_{j} t^{\gamma}\right)$ and the hazard function

$$
h_{j}\left(t, \boldsymbol{x}_{j}\right)=\alpha_{j} \gamma t^{\gamma-1}
$$

describes near-ground vortex demise for aircraft type $j$. Equation (4) coefficients are estimated through maximum likelihood, and the associated confidence intervals involve standard normal z-scores ${ }^{* *}{ }^{5}$ In other words, inference for Weibull regression models is no more complicated than inference for linear least-squares methods.

\section{The proportional hazards assumption}

The right side of Eq. (5) is the product of two functions: the Weibull shape parameter $g\left(\boldsymbol{x}_{j}\right)=\alpha_{j}$ which captures aircraft type-specific effects on vortex survival, and $h_{0}(t)=\gamma t^{\gamma-1}$. (And, $h_{0}(t)$ is the hazard function of a Weibull $(1, \gamma)$ random variable.) Weibull regression is a special case of 'proportional hazards' modeling ${ }^{5}$ because ratios of hazard functions are independent of time; they are strictly functions of characteristics for aircraft types $j$ and $k$ :

$$
\frac{h_{j}\left(t, \boldsymbol{x}_{j}\right)}{h_{k}\left(t, \boldsymbol{x}_{k}\right)}=\frac{g\left(\boldsymbol{x}_{j}\right)}{g\left(\boldsymbol{x}_{k}\right)}=\exp \left[-\frac{1}{\tau} \boldsymbol{\beta} \cdot\left(\boldsymbol{x}_{j}-\boldsymbol{x}_{k}\right)\right] .
$$

\section{E. Stratified models}

Weibull regression imposes an assumption that, between any two aircraft types, the relative risk of instantaneous vortex demise is always proportional (Eq. (6)). This assumption might not seem reasonable if a Weibull regression analysis is applied to disparate subjects; it might be inappropriate to assume the hazard functions describing B-737 and B-747 vortex demise are proportional. In such cases, the analyst defines categories of similar individuals (called 'strata') - so that Eq. (6) is reasonable within, not between, the strata - and fits a Weibull regression model to each. ${ }^{5}$

This enlarges the set of Weibull random variables needed to describe vortex demise over the entire observation set. Near-ground vortex survival probabilities for aircraft type $j$ belonging to strata $i$ is assumed $V_{i j} \sim$ Weibull $\left(\alpha_{i j}, \gamma_{i}\right)$. Note the shape parameters $\gamma_{i}=1 / \tau_{i}$ vary only between the strata. Aircraft-specific effects on vortex longevity are captured by the scale parameters $\alpha_{i j}=\exp \left[-\gamma_{i}\left(\beta_{0 i}+\boldsymbol{\beta}_{i} \cdot \boldsymbol{x}_{j}\right)\right]$. In stratified form, the Weibull regression technique described in section $C$ assumes the vortex decay process is described by a larger, categorized set of polynomial hazard functions

$$
h_{j i}\left(t, \boldsymbol{x}_{j}\right)=g_{i}\left(\boldsymbol{x}_{j}\right) \times h_{0 i}(t)=\alpha_{i j}\left(\gamma_{i} t^{\gamma_{i}-1}\right) .
$$

Weibull regression was performed with the LIFEREG procedure included in SAS software.

\section{Frankfurt Survival Data}

\section{A. Aircraft and their traits}

Weibull regression data comprises a set of 10,000 vortex lifetime measurements observed at Frankfurt am Main International Airport. This is the same data set ${ }^{7}$ the Volpe Center examined with a nonparametric regression method. ${ }^{\dagger \dagger}$ To remain consistent with the Frankfurt data set and related studies, V1 denotes the downwind vortex (the first vortex detected by the anemometer array); the upwind vortex (with respect to the crosswind) is denoted V2. Results for both categories are discussed, yet this paper focuses on V1 since it is more relevant to aircraft separation

Weibull form as $n \rightarrow \infty$. Moreover, vortex survival described by $V_{j} \sim$ Weibull $\left(\alpha_{j}, \gamma\right)$ implies $\ln V_{j} \sim \operatorname{evd}\left(\ln \alpha_{j}, 1 / \gamma\right)$, which is the logic behind $f(\varepsilon)$ from Eq. (1). ${ }^{5}$

${ }^{* *}$ Estimating a two-sided $100(1-\delta) \%$ confidence interval involves computing a $z$-score $Z_{\delta 2}$. Assuming

$Z \sim \operatorname{Normal}(0.1)$, the appropriate $z$-score is the value for which $P\left(Z>Z_{\delta / 2}\right)=\delta / 2$.

${ }^{++}$As the name suggests, nonparametric methods make no density assumptions. Those empirical results motivated us to consider certain densities, including the Weibull. 
standards at parallel runways. Two characteristics of each aircraft type $j$ were considered: wingspan $b_{j}$, and maximum landing weight $W_{j}$. Maximum landing weight was chosen as a proxy variable for the actual weight of the arriving aircraft. Two more variables were considered to test if wingspan and weight in combination influence vortex longevity (i.e., to test whether $V_{j}$ is a nonlinear function of $b_{j}$ and $W_{j}$ ). A statistically significant wing loading term, $L_{j}=W_{j} / b_{j}^{2}$, is evidence of an inverse and nonlinear relationship between the effects of wingspan and weight on vortex survival. A statistically significant product term $b_{j} \times W_{j}$, albeit contrived, provides evidence that (the effects of) wingspan and weight are somehow correlated.

This analysis was performed several years after Frankfurt measurements were collected (1997-1998). Care was taken to include only aircraft types in fleet service ${ }^{2}$ at that time to compute average values of $b_{j}$ and $W_{j}$. For example, average $b_{j}$ and $W_{j}$ for the B-737 type excludes the -700 series; it was not in service by 1998 . Table 1 lists the aircraft types considered with their respective wingspan $b_{j}$, maximum landing weight $W_{j}$ and wing loading $L_{j}$. (Values of the contrived term $b_{j} \times W_{j}$ are omitted.) Table 1 column 'Cases' tabulates the 10,000 sets of lifetime measurements (for V1 and V2) the anemometer array detected along either Frankfurt runway 25L or 25R.

Table 1 Aircraft types and dimensions

\begin{tabular}{ccccc}
\hline \hline & \multicolumn{4}{c}{ Large $^{\mathrm{a}}$} \\
Type $^{\mathrm{b}}(j)$ & $b_{i}$, meters & $W_{i}, 10^{4} \mathrm{~kg}$ & $L_{i}, 10^{4} \mathrm{~kg} / \mathrm{m}^{2}$ & Cases \\
\hline $\mathrm{A}-319$ & 34.1 & 30.379 & 261.257 & 472 \\
$\mathrm{~A}-320$ & 34.1 & 32.077 & 275.855 & 1552 \\
$\mathrm{~A}-321$ & 34.1 & 36.982 & 318.039 & 984 \\
$\mathrm{~B}-737$ & 28.9 & 29.889 & 357.858 & 2012 \\
$\mathrm{~B}-757$ & 38.0 & 46.296 & 320.611 & 228 \\
MD-80 & 32.9 & 29.387 & 272.201 & 411 \\
BAe-146 & 26.3 & 17.857 & 258.166 & 152 \\
& & Heavy & & \\
Type & $b_{j}$, meters & $W_{j}, 10^{4} \mathrm{~kg}$ & $L_{j}, 10^{4} \mathrm{~kg} / \mathrm{m}^{2}$ & Cases \\
A-300 & 44.8 & 66.579 & 331.725 & 703 \\
A-310 & 43.9 & 60.273 & 312.749 & 570 \\
A-330 & 60.3 & 89.665 & 246.597 & 41 \\
A-340 & 60.3 & 91.615 & 251.961 & 254 \\
B-747 & 62.1 & 143.794 & 373.472 & 1514 \\
B-767 & 49.7 & 67.460 & 273.109 & 599 \\
B-777 & 62.9 & 109.844 & 278.076 & 107 \\
DC-10 & 50.4 & 92.538 & 364.537 & 158 \\
L-1011 & 47.3 & 81.129 & 362.621 & 40 \\
MD-11 & 51.8 & 67.460 & 251.851 & 203 \\
\hline \hline
\end{tabular}

a. $18,600-115,700 \mathrm{~kg}$ maximum certificated gross takeoff weight (MCGTOW)

b. Aircraft Identification Code (AIC) Model.

c. Includes DC-9 type

d. $>115,700 \mathrm{~kg}$ MCGTOW

Table 1 also labels the two strata defined for the Weibull regression modeling. The strata mimics two categories of maximum certificated gross takeoff weight (MCGTOW). The U.S. categorizes aircraft with MCGTOW in excess of 255,000 pounds as 'Heavy' (henceforth subscripted as $i=1$ ) and aircraft with MCGTOW between 41,000 255,000 pounds $(18,600-115,700 \mathrm{~kg}$ ) as 'Large' (henceforth subscripted as $i=2){ }^{8}$. This study made one exception by including the B-757 in the Large category as opposed to its own category. Weibull regression is utilized to compare the effects different aircraft attributes have on vortex survival time. Weibull parameters $\alpha$ and $\gamma$ for the B757 should be estimated through one-sample hypothesis tests, not Weibull regression, if that aircraft is analyzed in isolation.

\section{B. Survival data assumptions}

For simplicity, a continuous time scale is assumed, even though vortex lifetimes at Frankfurt were measured in two-second intervals. Another important assumption is: vortex demise occurs precisely when reported. All measurements are assumed to be uncensored (in the parlance of survival data analysis). A censored subject corresponds to an unknown lifetime. In this context, censored measurements would occur whenever vortex longevity is known to exceed the tracking capabilities of the Frankfurt anemometer arrayr. Censoring occurs in 
several forms ${ }^{5}$, and any approach complicates analysis. An earlier report ${ }^{8}$ claimed it was impossible to tell which vortex lifetimes were censored so it seems reasonable to assume every recorded lifetime is exact.

\section{Results}

\section{A. Densities predicted by the regression method}

Results from a stratified Weibull regression analysis applied to V1 (downwind) vortex longevity along runway 25L are presented first. SAS software suppresses variables which are statistically insignificant (for this study, the criterion was a $p$-value $<0.05$ ), and so Table 2 relates the most parsimonious Weibull regression models. Per the equations expressed in section II.C, the variable coefficients dictate aircraft type-specific Weibull scale and shape parameters. The upper portion of Table 2 lists Eq. (4) estimated coefficients, their standard errors and confidence intervals derived from vortex demise data among aircraft in the 'Heavy' MCGTOW class. The lower portion lists those values derived from vortex demise data among 'Large’ MCGTOW class aircraft.

Table 2 Weibull parameters - V1 longevity along runway 25L.

\begin{tabular}{|c|c|c|c|c|c|c|}
\hline \multirow{3}{*}{ Parameter } & \multicolumn{4}{|c|}{ Heavy MCGTOW class } & \multirow{2}{*}{\multicolumn{2}{|c|}{$\begin{array}{l}\text { 95\% Confidence } \\
\text { Interval }\end{array}$}} \\
\hline & \multirow[t]{2}{*}{ Value } & \multirow[t]{2}{*}{$\begin{array}{l}\text { Standard } \\
\text { Error }\end{array}$} & \multirow[t]{2}{*}{$\begin{array}{c}\text { Test } \\
\text { Statistic }\end{array}$} & \multirow[t]{2}{*}{ p-value } & & \\
\hline & & & & & Lower & Upper \\
\hline$\beta_{0}$ & 4.356 & 0.037 & 13804.3 & $<0.0001$ & 4.283 & 4.429 \\
\hline$\beta_{b}$ & 0.007 & 0.001 & 99.15 & $<0.0001$ & 0.002 & 0.009 \\
\hline$\gamma$ & 3.642 & 0.053 & & & 3.540 & 3.747 \\
\hline & & Larg & CGTOW c & & & \\
\hline$\beta_{0}$ & 3.822 & 0.065 & 3455.01 & $<0.0001$ & 3.695 & 3.950 \\
\hline$\beta_{b}$ & 0.014 & 0.003 & 24.06 & $<0.0001$ & 0.009 & 0.020 \\
\hline$\beta_{W}$ & 0.004 & 0.002 & 4.57 & 0.0324 & 0.0003 & 0.008 \\
\hline$\gamma$ & 2.833 & 0.034 & & & 2.767 & 2.901 \\
\hline
\end{tabular}

For aircraft $j$ of the MCGTOW 'Heavy' class $(i=1)$, wingspan is statistically significant and not maximum landing weight nor the nonlinear terms. Weibull scale parameters estimated for aircraft $j$ are $\alpha_{1 j}=\exp \left[-3.642 \times\left(4.356+0.007 \cdot b_{j}\right)\right]=\exp \left(-15.865-0.025 \cdot b_{j}\right)$.

It follows from Eq. (7) that the set of hazard functions is

$$
h_{j 1}\left(t, \boldsymbol{x}_{j}\right)=\left(3.642 \cdot t^{2.642}\right) \times \alpha_{1 j}=\left(3.642 \cdot t^{2.642}\right) \times \exp \left(-15.865-0.025 \cdot b_{j}\right) .
$$

By contrast, for aircraft $j$ of the MCGTOW 'Large' class $(i=2)$, both wingspan and maximum landing weight are statistically significant. (Landing weight distinguishes between the three Airbus variants with identical wingspan.) Estimated Weibull scale parameters for aircraft in this category are $\alpha_{2 j}=\exp \left[-2.833 \times\left(3.822+0.014 \cdot b_{j}+0.004 \cdot W_{j}\right)\right]=\exp \left(-10.828-0.04 \cdot b_{j}-0.011 \cdot W_{j}\right)$.

The set of polynomial hazard functions from Eq. (7) is

$$
h_{j 2}\left(t, \boldsymbol{x}_{j}\right)=\left(2.833 \cdot t^{1.833}\right) \times \alpha_{2 j}=\left(2.833 \cdot t^{1.833}\right) \times \exp \left(-10.828-0.04 \cdot b_{j}-0.011 \cdot W_{j}\right) .
$$

Note that every aircraft type-specific Weibull scale parameter is a linear combination of wingspan and weight; nonlinear interaction terms $L_{j}$ or $b_{j} \times W_{j}$ are never statistically significant. ${ }^{\text {护 }}$ As time passes, scale parameters $\alpha_{i j}$ remain constant, so the hazard functions of Eqs. (8) and (9) resemble polynomials.

\section{B. Plotting and interpreting curves}

Figure 3a plots four functions associated with Heavy aircraft, and Fig. 3b does the same for four Large aircraft. Recall, LES suggests vortex strength declines even more rapidly beyond the linking time. Although the hazard functions of Fig. 3 are never expressed relative to any linking time, the curves for each aircraft type clearly suggest a similar decay process; the risk of instantaneous vortex demise increases in a nonlinear fashion as time passes. The

\footnotetext{
${ }^{\ddagger}$ Linear functions of wingspan and weight are evidence that variables $b_{j}$ and $W_{j}$ are independent in the strongest sense. A nonlinear term would indicate 'conditional independence' in which the pair of variables can only be assumed independent if survival analysis 'conditioned' on one of them (i.e., held it fixed).
} 
behavior of hazard functions as probability analogs to LES curves describing vortex strength was illustrated in Fig. 2.
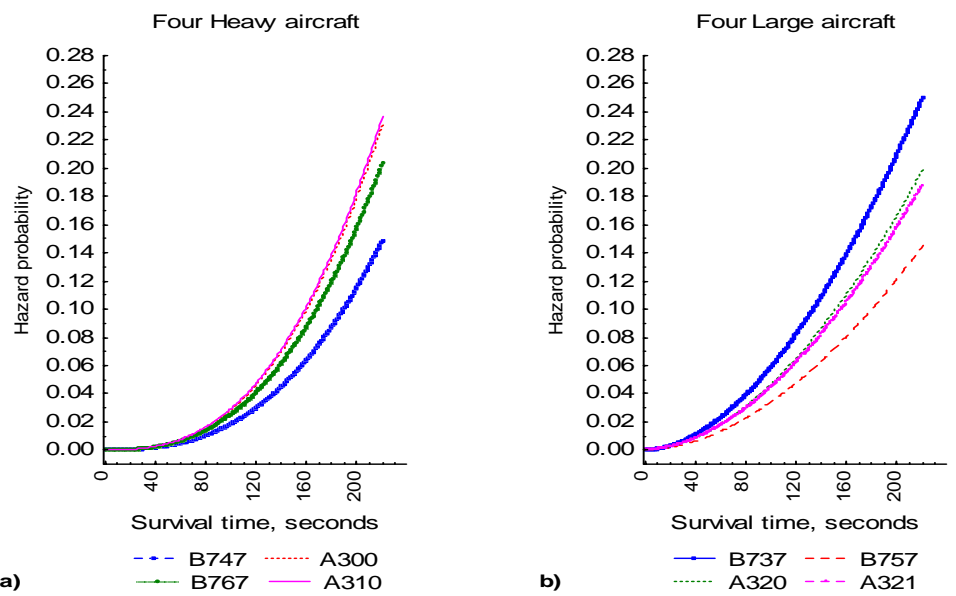

Figure 3. Polynomial hazard functions for Heavy (Fig. 3a) and Large aircraft (Fig. 3b).

Because near-ground vortices from Heavy aircraft generally survive longer than vortices from Large aircraft, the upward trend in hazard rates for Heavy aircraft (Fig. 3a), at $t=40$ seconds, occurs later than the analogous trend in hazard rates for Large aircraft vortices (Fig. 3b). Note that, between $0<t<40$ seconds, there is virtually zero chance of instant demise (given the vortex survives until $t$ ). For Heavy aircraft, Weibull scale parameters $\alpha_{1 j}=-15.865-0.025 \cdot b_{j}$ get smaller as wingspan increases, and densities all share Weibull shape parameter $\gamma_{1}=$ 3.642. The B-747 hazard function (Fig. 3a, rightmost curve) possesses the slowest rate of increase due to the aircraft's large wingspan. This is to say that, over time, B-747 V1 vortices always possess the smallest risk of instant demise (i.e., the greatest survival probabilities) among Heavy aircraft considered by this assessment.

By contrast, the B-737 hazard function (Fig. 3b, leftmost curve) is steepest; B-737 near-ground V1 - relative to the other three aircraft - always have the highest risk of instant demise. All densities describing Large aircraft V1 lifetimes share Weibull shape parameter $\gamma_{2}=2.833$. Lighter weight and smaller wingspan each reduce Weibull scale parameters $\alpha_{2 j}=-15.865-0.04 \cdot b_{j}-0.011 \cdot W_{j}$, and the B-737 possesses the smallest of each (Table 1). From

Eq. (6) curves within, but not between, Figs. 3a and 3b are assumed proportional to each other. Both charts indicate hazard probabilities (the $y$-axis) range between 0.12-0.22 (12-22\%) among near-ground V1surviving at least $t=200$ seconds (the $x$-axis). It is very unlikely that near-ground V1 (traveling from runway 25L) would survive until $t=$ 200 seconds, regardless of aircraft type, and so hazard functions eventually overlap (i.e., it becomes difficult to discriminate vortex demise rates by aircraft type, for large $t$ ).

Ratios of hazard functions estimate the relative risk of instant vortex demise between any two aircraft in the same weight class, expressed as a probability. A comparison of two Heavy aircraft, the A-310 and the B-747, illustrates model inference. Table 2 (upper half) estimates the Weibull shape parameter $\gamma_{1}=1 / \tau_{1}=3.642$ as well as the wingspan coefficient $\beta_{b}=0.007$. (Dimensions for both aircraft are presented in Table 1.) The hazard ratio Eq. (8) is $\exp (-0.025 \cdot(43.9-62.1))=1.576$. Throughout the $\mathrm{V} 1$ aging process, those from the A-310 are predicted to have a $58 \%$ higher probability of instantaneous demise than B-747 vortices V1, attributable to the difference in wingspan for those aircraft. In Fig. 2a the A-310 hazard (leftmost) curve is always 1.576 times higher than the B747 (rightmost) hazard curve.

To compare the A-320 with the B-737, Table 2 (lower half) estimates the Weibull shape parameter $\gamma_{2}=1 / \tau_{2}=$ 2.833, the coefficient for wingspan $\beta_{b}=0.014$, and coefficient for weight $\beta_{W}=0.004$. (Again, dimensions are presented in Table 1.) The hazard ratio is $\exp (-0.04 \cdot(28.9-34.1)-0.011 \cdot(29.889-32.077))=1.261$. Throughout the V1 aging process, A-320 vortices are predicted to have a 26\% higher probability of instant demise than B-737 vortices. This prediction is attributable to differences in both wingspan and maximum landing weight. In Fig. 3b the B-737 (leftmost) curve is always 1.261 times higher than the adjacent A-320 curve. 
To evaluate prediction accuracy it is easier to examine Weibull survival functions $S(t)$ rather than hazard functions $h(t)$. The stratified Weibull method yields a set of survival functions, each defined by a pair of estimated shape and scale parameters:

$$
S_{j i}\left(t, \mathbf{x}_{j}\right)=\exp \left(-\alpha_{i j} t^{\gamma_{i}}\right)
$$

For near-ground vortices V1 from runway 25L, Fig. 4 plots Eq. (10) survival functions (dark curves) for the B737, A-320, A-310, and B-747.

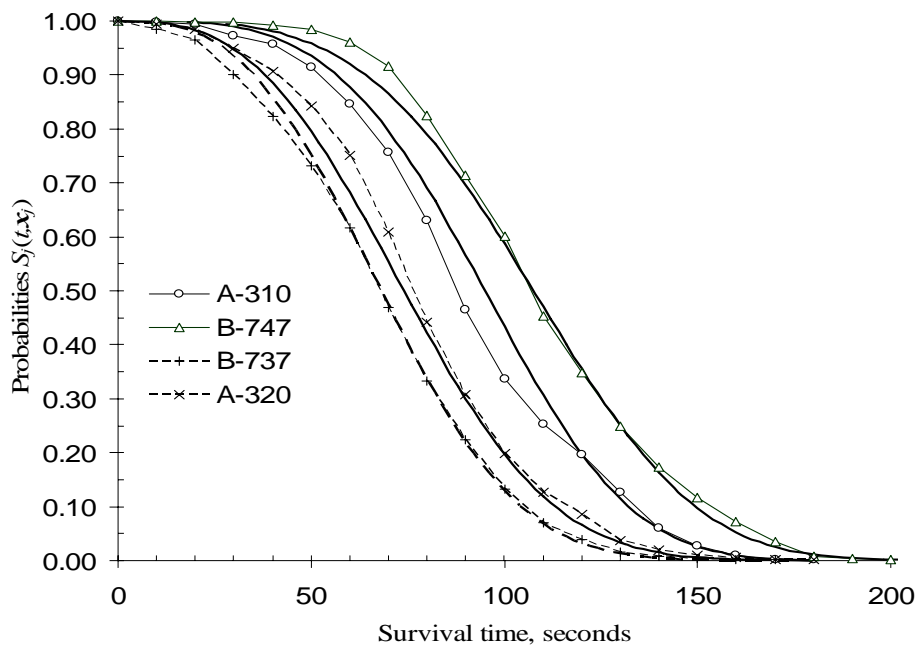

Figure 4. Predicted survival functions of four aircraft.

Figure 4 also plots the four survival functions empirically estimated from Frankfurt data (marked curves). Marked curves plot the percentage of vortices, downwind of runway 25L, surviving longer than $t=0,10,20, \ldots, 200$ seconds since the aircraft passed over the anemometer array. Predictions for B-737 and B-747 vortex lifetime probabilities agree particularly well with Frankfurt measurements. The Weibull density underestimates A-320 vortex survival probabilities $S(t)$ for $50 \leq t \leq 70$ seconds, but is otherwise quite accurate. The density consistently overestimates A-310 vortex survival probabilities.

Confidence intervals around each survival function are easily derived. The last two columns of Table 2 present the values needed to draw the two survival curves representing the lower and upper bound, respectively, at the 95\% level. To reduce clutter Fig. 5 presents estimated survival functions (solid curves), their respective confidence intervals (dashed bands), and observed proportions (markers) for only the B-737 and B-747. (Confidence intervals are drawn between the $5^{\text {th }}$ and $95^{\text {th }}$ percentiles for vortex lifetime. That is, $t$ spans the range values beginning with $S(t)=0.05$ up through $S(t)=0.95$.)

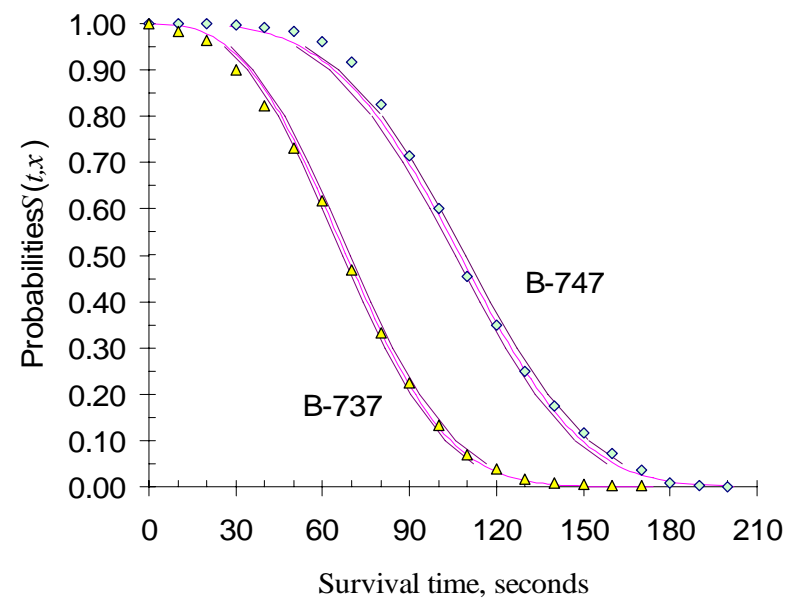

Figure 5. Tight 95\% confidence intervals surround predicted survival probabilities. 
Regression goodness-of-fit is sufficiently strong so that, in general, confidence intervals have the desirable property that the bounds remain tight throughout the vortex aging process. Plotting densities (Fig. 6) is simply a matter of drawing Eq. (2) for each pair of aircraft type-specific Weibull shape and scale parameters.

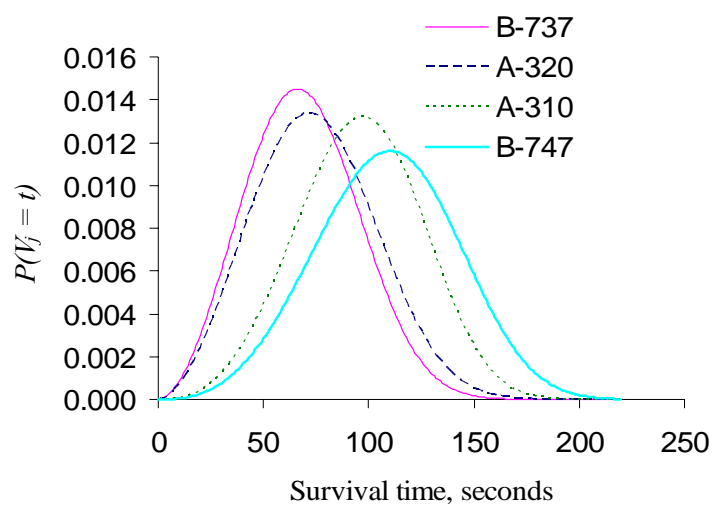

Figure 6. Probability densities of downwind vortex survival - four aircraft approaching runway 25L.

Densities for V1 survival probabilities are asymmetric; densities for the two Large aircraft have elongated righthand tails, and elongated left-hand tails for the pair of Heavy aircraft. Note that the highest points of each density (the modes), corresponding to the most probable vortex lifetime for the respective aircraft, shift successively towards the right (longer lifetimes) with increases in either wingspan or maximum landing weight (see Table 1).

\section{Residuals analysis}

Typically, a complete regression analysis assesses residuals (the Eq. (4) random error terms). Weibull regression assumes residuals that are not normally distributed (see footnote, p. 4). The Cox-Snell method confirms how closely residuals follow the assumed random process and, by extension, whether Weibull densities are reasonable for estimating vortex lifetimes. ${ }^{5}$ Stratified Weibull regression generates a set of vectors $\boldsymbol{r}_{\boldsymbol{j} i}$ each associated with each aircraft type $j$ of MCGTOW classes $i=1,2$. According to the Cox-Snell method, a specific $\boldsymbol{r}_{j i}$ scatterplot will follow a 45-degree line through the origin if Weibull regression assumptions are met. ${ }^{\S \S}$ Two scatterplots depicting Heavy (Fig. 7a) and Large (Fig. 7b) residuals pass through the origin and fall along the line $y=x$.
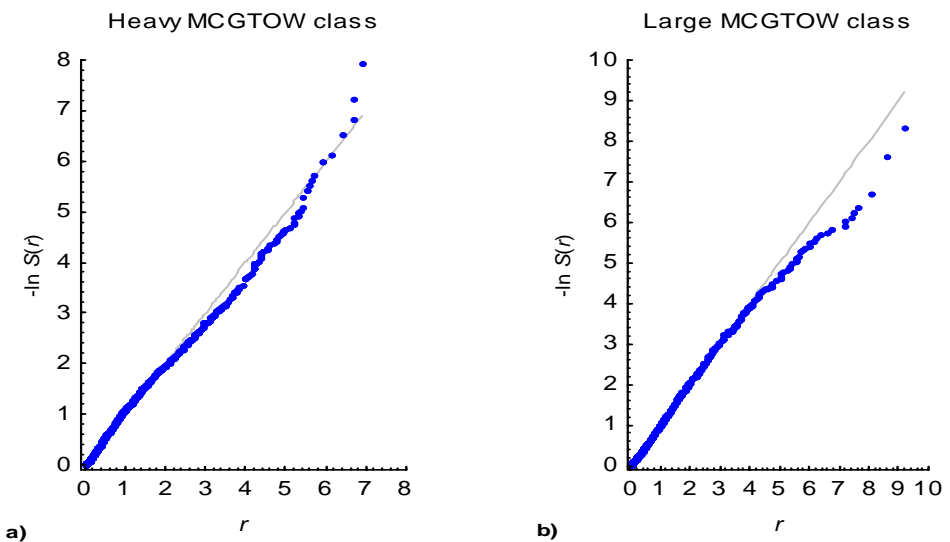

Figure 7. Weibull density functions are adequate for modeling vortex demise (downwind of runway 25L).

Predicting runway 25L V1 longevity probabilities with a set of aircraft type-specific Weibull density functions appears quite appropriate. The modest departure of Fig. 7b residuals corresponds to very improbable V1 lifetimes among Large aircraft (recall, the B-757 is analyzed as a Large aircraft). Unfortunately, a weakness of Cox-Snell analysis is that scatterplots cannot be used to detect bias in the Weibull densities. ${ }^{5}$ That is from Fig. $7 \mathrm{~b}$, when

${ }_{\S}^{\S}$ The key property behind Cox-Snell analysis is $\boldsymbol{r}_{j i} \sim$ Exponential(1) when assumptions hold, which implies $S\left(\boldsymbol{r}_{j i}\right)=\exp \left(-\boldsymbol{r}_{j i}\right)$. The scatterplot simply graphs points $\left[\boldsymbol{r}_{j i},-\ln \mathrm{S}\left(\boldsymbol{r}_{j i}\right)\right]=\left(\boldsymbol{r}_{j i}, \boldsymbol{r}_{j i}\right)$ for adequate models. 
residuals fall below the reference line, it is impossible to infer whether Eqs. (2) consistently under-estimate (or overestimate) Large aircraftV1 longevity probabilities late in the decay process.

\section{Exploring a Constant Weibull Shape Parameter}

A set of Weibull densities possessing aircraft type-specific scale parameters predict vortex V1 survival probabilities quite well (see Eqs. (8) and (9)). These densities possess one of two shape parameters since longevity measurements are divided into two classes based upon aircraft MCGTOW. The best-fit shape parameters $\gamma_{1}=3.642$ $>3.0$ (for the Heavy class) and $\gamma_{2}=2.833<3.0$ (for the Large class), so the analysis examined if a fixed $\gamma \equiv 3.0$ would yield comparable predictions. Repeating the analysis with $\gamma \equiv 3.0$ (Table 3) has virtually no effect on the coefficients or statistical significance of wingspan and weight.

Table 3 Weibull regression fit when parameter $\gamma=3.0$.

\begin{tabular}{|c|c|c|c|c|c|c|}
\hline \multirow{3}{*}{ Parameter } & \multicolumn{4}{|c|}{ Heavy MCGTOW class } & \multirow{2}{*}{\multicolumn{2}{|c|}{$\begin{array}{l}\text { 95\% Confidence } \\
\text { Interval }\end{array}$}} \\
\hline & \multirow[t]{2}{*}{ Value } & \multirow[t]{2}{*}{$\begin{array}{l}\text { Standard } \\
\text { Error }\end{array}$} & \multirow[t]{2}{*}{$\begin{array}{r}\text { Test } \\
\text { Statistic }\end{array}$} & \multirow[t]{2}{*}{ p-value } & & \\
\hline & & & & & Lower & Upper \\
\hline$\overline{\beta_{0}}$ & 4.321 & 0.045 & 9274.35 & $<0.0001$ & 4.233 & 4.408 \\
\hline$\beta_{b}$ & 0.007 & 0.001 & 72.17 & $<0.0001$ & 0.001 & 0.009 \\
\hline$\gamma$ & 3.0 & & & & & \\
\hline \multicolumn{7}{|c|}{ Large MCGTOW class } \\
\hline$\beta_{0}$ & 3.838 & 0.061 & 3911.67 & $<0.0001$ & 3.717 & 3.958 \\
\hline$\beta_{b}$ & 0.014 & 0.003 & 26.50 & $<0.0001$ & 0.009 & 0.019 \\
\hline$\beta_{W}$ & 0.004 & 0.002 & 4.91 & 0.027 & 0.0005 & 0.008 \\
\hline$\gamma$ & 3.0 & & & & & \\
\hline
\end{tabular}

Recall $h_{j i}\left(t, \boldsymbol{x}_{j}\right)=\alpha_{i j}\left(2 t^{\gamma-1}\right)$ so that $\gamma=3.0$ assumes vortex demise is a random process described by quadratic hazard functions, regardless of aircraft wingspan and weight (and MCGTOW class). This family of Weibull densities predicts virtually identical survival probabilities as the set estimated by Table 2 parameters. Yet, there is a tradeoff between accuracy and simplicity, particularly among Heavy class aircraft. Figure 8a illustrates that the B747 probability density with parameter $\gamma_{1}=3.642$ (Table 2, top) appears to agree more closely with the empirical survival function than a density with parameter $\gamma=3.0$. For the A-310 the density with parameter $\gamma=3.0$ appears more accurate, while a density with $\gamma_{1}=3.642$ consistently overestimates probabilities (Fig. 8b). Both models sandwich the data set for shorter lifetimes (the $\gamma=3.0$ density is more accurate and less biased), then cross at roughly $t=120$ seconds, and afterward the $\gamma_{1}=3.642$ density is more accurate (now, the $\gamma=3.0$ model is biased).
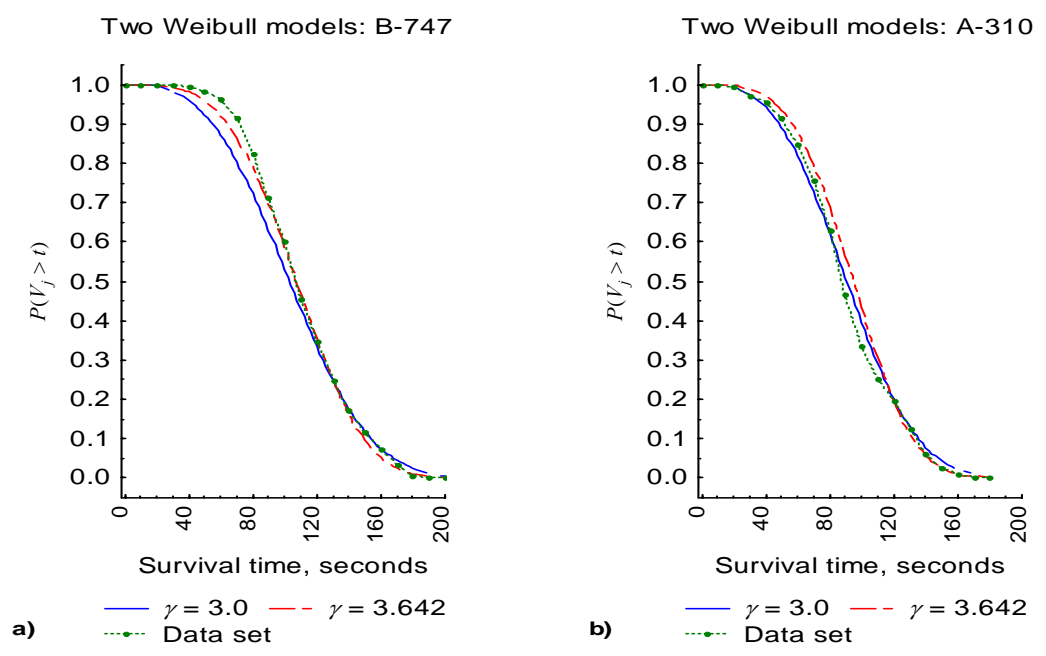

Figure 8. Two examples - fixing $\gamma=3.0$ involves a tradeoff between simplicity and accuracy. 
To assume V1 demise as a random process described by quadratic hazard functions $(\gamma=3.0)$ might be reasonable for any aircraft type, but more analyses (ideally on data from several airports) are recommended. The preceding analysis only considered V1 vortices traveling from Frankfurt runway 25L toward runway 25R. (These instances comprise the majority of cases in the Frankfurt data set.)

\section{Concluding remarks}

Regression analyses assume best-fit equation residuals (differences between predicted and actual values) follow a specific random process. So, the density form describing residuals will dictate the density form describing V1 survival probabilities. (The Appendix describes some of the authors' lessons learned between this and earlier modeling attempts.) Aircraft wingspan in particular appears useful in equations that estimate scale parameters for a family of Weibull distributions; diagnostics also suggest the random process controlling residuals is consistent with the assumed Weibull density form. (Weight is also useful to distinguish the 'Large' aircraft with identical wingspan.) For modeling vortex demise as a random process, the most important insight from the regression equations and diagnostic tests based upon Frankfurt V1 decay is that the proportional hazards assumption is confirmed. Between any two aircraft of the same weight class, the rates at which their respective V1 vortices die out remain in constant proportion over time. Among 'Heavy' aircraft, that proportion is determined by the ratio of the two wingspans.

\section{Appendix}

In an earlier paper ${ }^{3}$, several graphs were presented as evidence for $S(t) \propto \exp \left(-\beta t^{2}\right)$ suggesting near-ground vortex longevity ${ }^{* * *}$ could be modeled as $V \sim$ Weibull $(\beta, 2)$ random variables, corresponding to a linear hazard functions: $h(t)=\beta t^{(2-1)}=\beta t$ (see section II, part B). Applied to current Frankfurt data, Fig. 9 reproduces the simple linear regression equation predicting demise rates for V1 vortices traveling away from runway 25L.

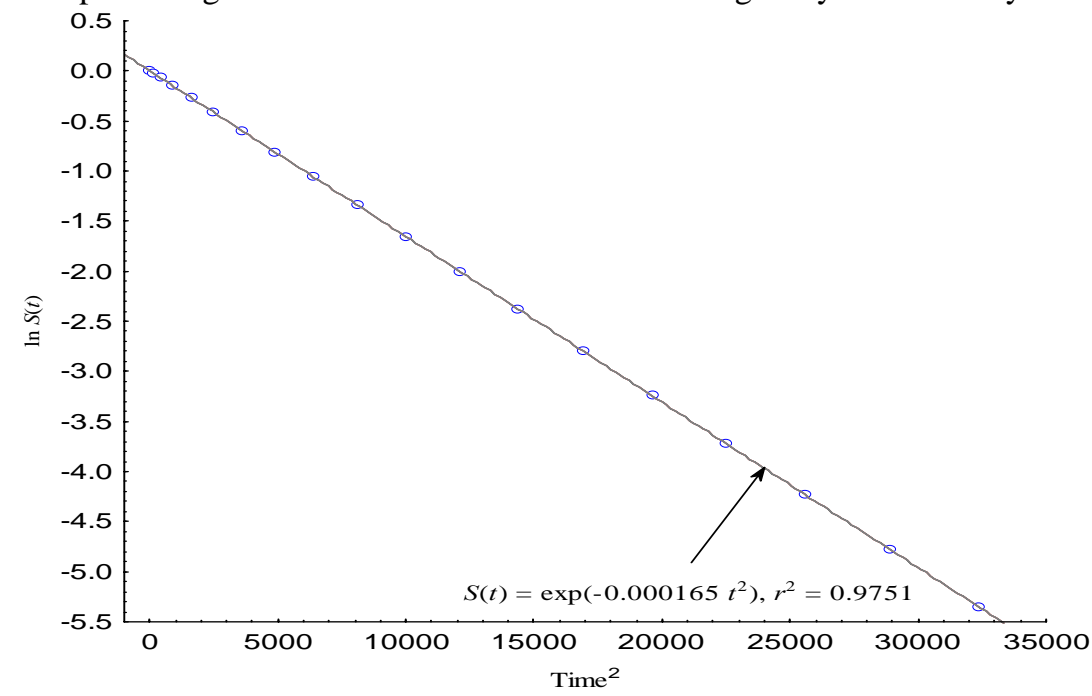

Figure 9. Linear least-squares regression assumed a linear hazard function $(\gamma=2)$ for V1 decay.

That linear least-squares analyis ${ }^{3}$ fitted lines predicting survival $S(t)$ as a function of one independent variable, $t$ (which was also reported in ten second intervals):

$$
\ln [S(t)]=\beta_{0}+\beta_{1} t^{2}+\varepsilon
$$

The fit of the example equation $\ln [S(t)]=-0.000165 t^{2}$ is excellent $\left(r^{2}=0.975\right)$, but the regression assumption $\varepsilon^{\sim} \operatorname{Normal}(0,1)$ is implausible. One piece of evidence is the normal probability plot for Fig. 9 residuals, which are not randomly scattered near the reference line (Fig. 10).

${ }^{* * * *}$ At that time, predictions were not aircraft-specific, so b is a universal parameter. Further, the only data set available was half its present size, comprising 5,000 measurements. 


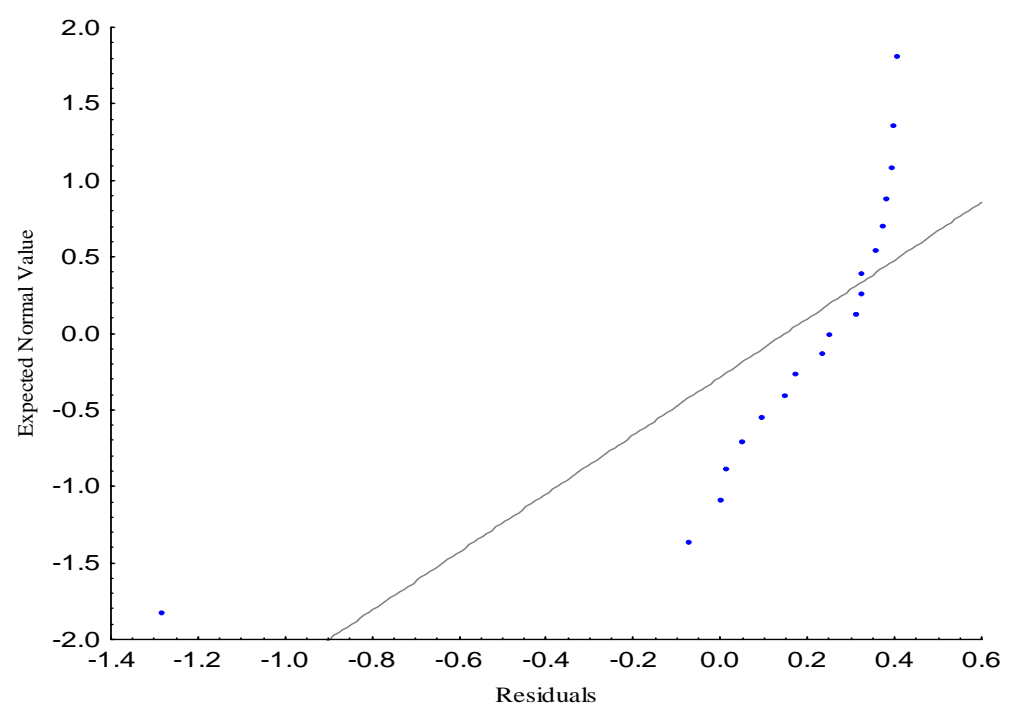

Figure 10. The random error terms appear non-Normal, contradicting a least squares assumption.

The residuals not only deviate from the line, but note that, along the $x$-axis of Fig. 10, virtually all of them are positive. The best fit line from Fig. 9 would consistently overestimate V1 survival probabilities; in other words, Eq. (A-1) predictions were biased. Of the original 5,000 measurements, only 2,800 corresponded to V1 traveling from runway 25L. For Weibull regression analysis, 6,900 V1 measurements are available (out of 10,000 total). For illustration purposes only (since Weibull regression is preferred) the following describes the dramatic effects a) the larger set and b) aggregating survival data in ten-second intervals have on the validity of Eq. (A-1).

A least-squares line corresponding to $S(t) \propto \exp \left(-\beta t^{3}\right)$ has superior fit $\left(r^{2}=0.995\right)$ compared with Fig. 9 . However, once again $\varepsilon^{\sim \operatorname{Normal}}(0,1)$ imputes the wrong random process on V1 decay. Figure 11 includes three graphs; the best-fit line assuming $\gamma=3$ (top); the normal probability plot (center); a plot of predicted $S(t)$ versus the residuals (bottom). Residuals in the normal probability plot do not deviate as severely from the reference line, and they are relatively symmetric around $x=0$. Yet, $\varepsilon^{\sim \operatorname{Normal}}(0,1)$ is still implausible as the residuals are not randomly scattered about the solid reference line (bottom plot). If linear least-squares was appropriate and $\varepsilon^{\sim N o r m a l}(0,1)$ then the bottom scatterplot would resemble a random, symmetric 'cloud' of points. Linear least-squares is not an ideal technique for predicting $S(t)$, yet it does offer evidence for choosing $\gamma=3$ over $\gamma=2$.
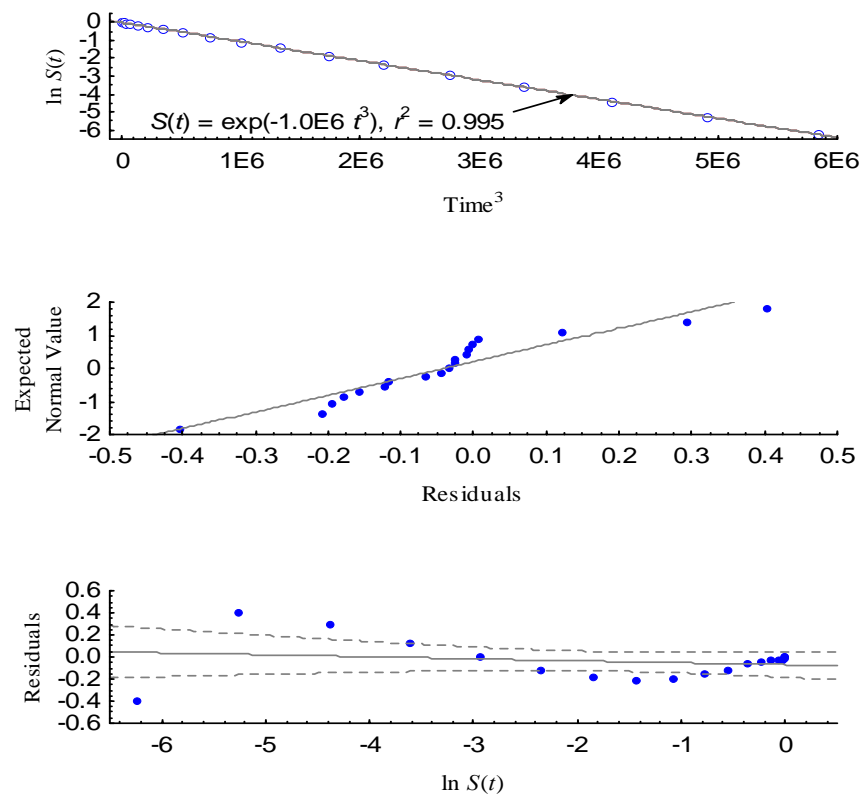

Figure 11. $S(t) \propto \exp \left(-\beta t^{3}\right)$ improves fit, but $\varepsilon^{\sim N o r m a l}(0,1)$ is the wrong random process on $\mathrm{V} 1$ decay. 
Fitting linear least-squares equations to values of $t$ and $S(t)$ computed every ten seconds, not every two seconds in the manner consistent with Frankfurt experiments, was another source of prediction error. ${ }^{3}$ A probability plot used in survival analysis illustrates how aggregating data affected $\gamma$ estimates and, in particular, demonstrates $V \sim$ Weibull $(\alpha, \underline{3})$ would have been a better assumption. ${ }^{\text {t† }}$

The cumulative hazard function $H(t)=\int_{0}^{t} h(x) d x$ mimics the relationship between a probability density $f(t)$ and the cumulative distribution $F(t)$. The Eq. (3) hazard function $h(t)$ implies $H(t)=\beta t^{\gamma}$ if $V \sim \operatorname{Weibull}(\beta, \gamma)$. Taking logarithms of both sides then isolating $t$ implies a linear relationship:

$$
\ln t=\frac{1}{\gamma} \ln \frac{1}{\beta}+\frac{1}{\gamma} \ln H(t)
$$

Both Weibull parameters can be estimated directly from Frankfurt data by fitting Eq. (A-2) and the (reciprocal of the) slope of that line estimates the shape parameter $\gamma$. The function $H(t)$ is estimated numerically following a multi-step algorithm. ${ }^{4}$

The best-fit line to data of the original assessment $(2,800 \mathrm{~V} 1$ measurements at runway $25 \mathrm{~L}$, aggregated in ten second intervals) is $\ln t=4.1535+0.5369 \cdot \ln H(t)$. So, estimated $\gamma$ is

$$
\gamma=\frac{1}{0.5369}=1.86
$$

This is certainly consistent with $V \sim \operatorname{Weibull}(\beta$, 2). Yet, if Eq. (A-2) is fitted with $t$ and $H(t)$ measured every two seconds, the line changes to $\ln t=4.3786+0.4016 \cdot \ln H(t)$. Now,

$$
\gamma=\frac{1}{0.4016}=2.49 \text {. }
$$

Thus, if V1 runway 25L survival data is evaluated consistently with the Frankfurt experiment, the validity of $V \sim$ Weibull $(\alpha, 2)$ is less clear.

Finally, Weibull regression data set (6,900 V1 measurements, in two second intervals, at runway 25L) yields the straight line $\ln t=4.5420+0.3445 \cdot \ln H(t)$, and this time

$$
\gamma=\frac{1}{0.3445}=2.90 \text {. }
$$

Once again, there is stronger evidence that $V \sim \operatorname{Weibull}(\beta, \underline{3})$; demise rates of $\mathrm{V} 1$ vortices (from runway 25L) - in this context aircraft type is immaterial - follow a random process described by a quadratic hazard function. Figure 12 summarizes the progressive effects on shape parameter $\gamma$. It plots three sets of points $[\ln (t), \ln H(t)]$ and the three fitted lines: a) on the right, the line estimating $\gamma=1.86$, b) in the center, the line estimating $\gamma=2.49$ and c) on the left, the line estimating $\gamma=2.9$.

${ }^{+\dagger \dagger}$ In the context of this discussion, there is one survival data set describing V1 longevity from runway 25L, which requires only one pair of Weibull parameters. The Weibull regression analysis estimated an entire set of densities, such that a) shape parameters $\gamma$ are MCGTOW class-specific and b) scale parameters $\alpha$ are aircraft type-specific. 


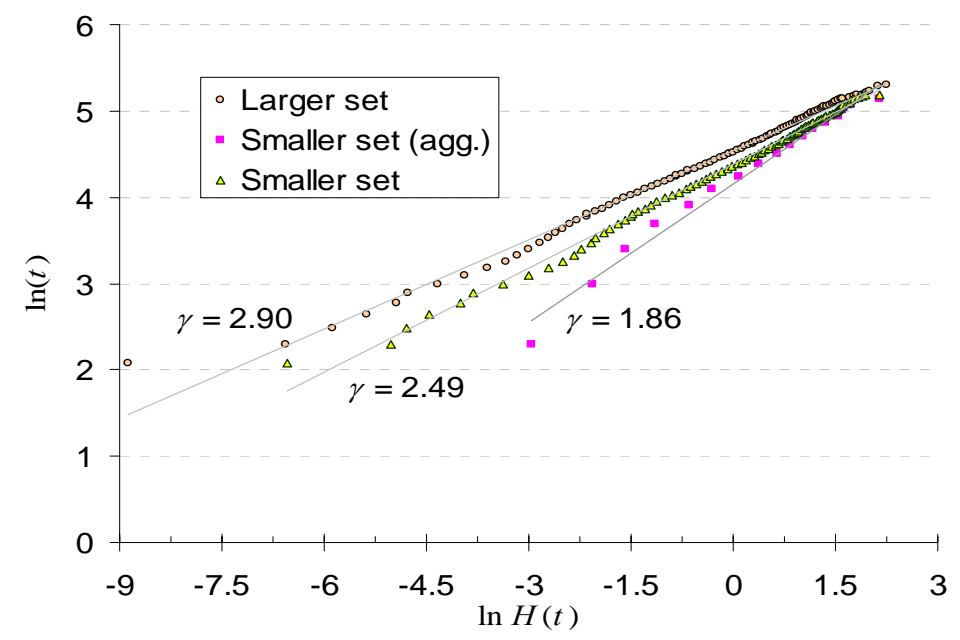

Figure 12. More survival data, evaluated in two second intervals, suggests $\gamma=3$, and not $\gamma=2$.

\section{References}

${ }^{1}$ Hallock, J. H., Osgood, S. P. and Konopka, J., “Wake Vortex Effects on Parallel Runway Operations”, AIAA2003-379, 2003.

${ }^{2} 2004$ Aerospace Source Book (2004), Aviation Week and Space Technology, 160, 3, pp. 66-71.

${ }^{3}$ Holzäpfel, F., “Probabilistic Two-Phase Wake Vortex Decay and Transport Model”, Journal of Aircraft, Vol. 40, No. 2, 2003, pp. 323 - 331.

${ }^{4}$ Proctor, F. H. and Switzer, G.F., "Numerical Simulation of Aircraft Trailing Vortices”, 9th Conf. On Aviation, Range and Aerospace Meteorology, Paper 7.12, American Meteorology Society, Orlando, Florida, Sept. 2000, pp. 511-516.

${ }^{5}$ Lee, E.T. and Wang, J.W., Statistical Methods for Survival Data Analysis, 3rd. ed., Wiley, Hoboken, 2003.

${ }^{6}$ NIST/SEMATECH e-Handbook of Statistical Methods. Internet WWW pages at URL: http://www.itl.nist.gov/div898/handbook/apr/section1/apr163.htm.

${ }^{7}$ Berk, L., "Predicting near-ground vortex lifetimes using survival analysis methods", DOT-VNTSC-FA27-PM05-15, U.S. DOT Volpe Center, 2005.

${ }^{8}$ Burnham, D.C., Hallock, J.N. and Greene, G.C., "Wake Turbulence Limits on Paired Approaches to Parallel Runways,” Journal of Aircraft, Vol. 39, No. 4, 2002, pp. 630 - 637. 